\title{
Study of the role of Mce3R on the transcription of mce genes of
} Mycobacterium tuberculosis

\author{
María P Santangelo ${ }^{1}$, Federico C Blanco ${ }^{1}$, María V Bianco ${ }^{1}$, Laura I Klepp ${ }^{1}$, \\ Osvaldo Zabal ${ }^{2}$, Angel A Cataldi ${ }^{1}$ and Fabiana Bigi*1
}

Address: ${ }^{1}$ Institute of Biotechnology, CICVyA-INTA, Los Reseros y Las Cabañas, 1712 Castelar, Argentina and ${ }^{2}$ Institute of Virology, CICVyA-INTA, Castelar, Argentina

Email: María P Santangelo - psantangelo@cnia.inta.gov.ar; Federico C Blanco - fblanco@cnia.inta.gov.ar;

María V Bianco - mbianco@cnia.inta.gov.ar; Laura I Klepp - lklepp@cnia.inta.gov.ar; Osvaldo Zabal - ozabal@cnia.inta.gov.ar;

Angel A Cataldi - acataldi@cnia.inta.gov.ar; Fabiana Bigi* - fbigi@cnia.inta.gov.ar

* Corresponding author

Published: 27 February 2008

BMC Microbiology 2008, 8:38 doi:10.1/86/147|-2180-8-38
Received: 14 November 2007

Accepted: 27 February 2008

This article is available from: http://www.biomedcentral.com/I47I-2180/8/38

(c) 2008 Santangelo et al; licensee BioMed Central Ltd.

This is an Open Access article distributed under the terms of the Creative Commons Attribution License (http://creativecommons.org/licenses/by/2.0), which permits unrestricted use, distribution, and reproduction in any medium, provided the original work is properly cited.

\begin{abstract}
Background: mce 3 is one of the four virulence-related mce operons of Mycobacterium tuberculosis. In a previous work we showed that the overexpression of Mce3R in Mycobacterium smegmatis and M. tuberculosis abolishes the expression of lacZ fused to the mce3 promoter, indicating that Mce3R represses mce3 transcription.
\end{abstract}

Results: We obtained a knockout mutant strain of $M$. tuberculosis $\mathrm{H} 37 \mathrm{Rv}$ by inserting a hygromycin cassette into the mce $3 R$ gene. The mutation results in a significant increase in the expression of mce3 genes either in vitro or in a murine cell macrophages line as it was determined using promoterlacZ fusions in M. tuberculosis. The abundance of mcel, mce 2 and mce 4 mRNAs was not affected by this mutation as it was demonstrated by quantitative RT-PCR. The mce3R promoter activity in the presence of Mce3R was significantly reduced compared with that in the absence of the regulator, during the in vitro culture of $M$. tuberculosis.

Conclusion: Mce3R repress the transcription of mce 3 operon and self regulates its own expression but does not affect the transcription of mce $I$, mce 2 and mce 4 operons of $M$. tuberculosis.

\section{Background}

Tuberculosis (TB), a chronic illness caused by Mycobacterium tuberculosis, is still a major worldwide disease. Pathogenic mycobacteria species have demonstrated a remarkable ability to survive in diverse conditions encountered during the infection process. However, even after decades of investigation, there is still little knowledge about mycobacterial pathogenesis. Understanding the infective process at the molecular and cellular levels will lead to new strategies to control this disease and even to the development of an effective vaccine.
The analysis of the complete sequence of the M. tuberculosis H37Rv genome revealed the presence of four paralogous mce genes, all encoded in an operon structure consisting of eight genes [1]. The biological function of Mce proteins is not known, but increasing evidence has demonstrated that they are clearly related to the virulence of Mycobacterium tuberculosis complex species [2-8].

Gene regulation is considered to play a central role in host-microbe interactions, and many virulence genes are regulated in response to the host. Casali and collaborators 


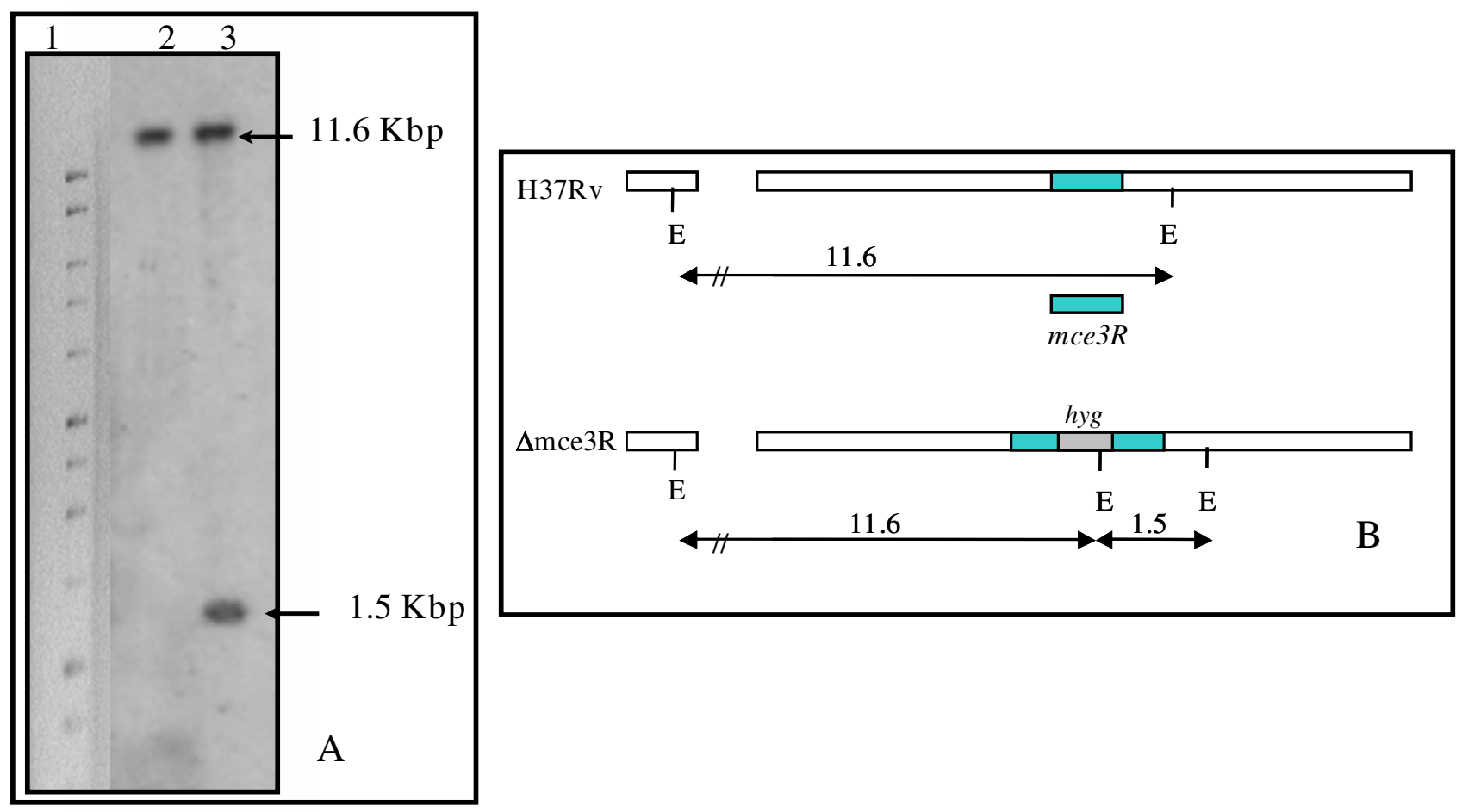

\section{Figure I}

Disruption of the mce3R gene of $M$. tuberculosis H37Rv. (A) Southern blot analysis of chromosomal DNA from sucR counterselected tuberculosis clone (lane 3) and parental strain (lane 2). Genomic DNA was digested with EcoRI and hybridized to the mce3R probe. Arrows indicate position of hybridizing fragments. MWM I kb Promega is shown on the left (lane I). (B) Restriction map of mutant and wild type strains. The insertion of hygromycin-resistant cassettes is indicated (hyg). Arrows represent the length of expected bands after digestion with EcoRI $(\mathrm{E})$. Value on each arrow indicates the molecular weight of expected bands expressed in base pairs (bp).

[9] identified a regulatory mechanism which controls mce1 expression. They have demonstrated that a homologue of the FadR subfamily of GntR transcriptional regulators, $\mathrm{Rv0165c}$ (designated Mce1R), is a negative regulator that intracellularly represses expression of the mce 1 operon. In addition, a gene encoding a putative transcriptional factor, Rv0586, is located immediately upstream of $m c e 2$ operon and it is transcribed in the same direction as that of $m c e 2$ genes. Furthermore, it has been found that there are growth phase and tissue specific differences in the expression of mce operons in M. tuberculosis [10-12] which is in agreement with the presence of regulatory mechanisms controlling mce transcription. In a previous work, we found evidence indicating that $M c e 3 R$, a TetR family transcriptional regulator, down-regulates the mce3 operon during the in vitro growing of M. tuberculosis [13]. We have demonstrated that the overexpression of Mce3R in both M. smegmatis and M. tuberculosis abolishes the expression of a gene reporter fused to mce3 promoter.
TetR family members often regulate their own synthesis [14-18]. The classic example of self regulation in members of this family protein is a repressor involved in resistance to tetracycline of Escherichia coli, which has given the name TetR to the group [19]. In a number of TetR-autoregulated systems the regulator and the structural genes are divergently transcribed and the region for protein binding overlaps the promoters placed in the intergenic region $[15,18,20]$. That is the case of mce3R, which is placed upstream of mce3 operon, oriented in the opposite direction and separated from it by a region of $880 \mathrm{bp}$.

In this work we validate the role of Mce3R in repressing the mce 3 transcription in $M$. tuberculosis by analyzing gene expression in a mce3R-knockout $M$. tuberculosis strain. We also found that this regulation is exclusive for the mce 3 operon among mce genes and that the Mce3R repressor regulates its own expression. 


\section{Results \\ Construction of a mce3R mutant in $M$. tuberculosis}

As a first step to assess the mce 3 operon expression in the absence of Mce3R, we obtained a knockout mutant strain of $M$. tuberculosis H37Rv by inserting a hygromycin cassette into the mce3R gene. The site-directed mutant strain of $M$. tuberculosis was obtained by two-step mutagenesis strategy by using the p2NIL shuttle plasmid [21], which carries the lac $Z$ gene and the counter selectable marker $s a c B$. Allelic exchange was confirmed in the selected clones $\left(\mathrm{Hy}^{\mathrm{R}}, \mathrm{Km}^{\mathrm{S}}\right.$, and $\mathrm{Sac}^{\mathrm{R}}$ ) by Southern blotting (Fig. 1A), since the mutant showed a hybridizing fragment of about $1.5 \mathrm{~kb}$ absent in the wild-type strain. This polymorphism is due to the introduction of an extra EcoRI site present in the hygromycin cassette (Fig. 1B). The mutant strain was designated $\Delta m c e 3 R$. The mutation was complemented by transforming the plasmid pSummce3R into the mutant.

\section{In vitro characterization of $\Delta m c e 3 R$}

To determine whether $m c e 3 R$ disruption introduces alterations during in vitro growth, growth curves of the $\Delta m c e 3 R$ mutant, complemented, and parental strains were compared under standard culture conditions. All assayed strains showed similar doubling time and growth characteristics throughout the culture period (Fig. 2). This result indicates that the mutation does not affect the in vitro growth of $M$. tuberculosis.

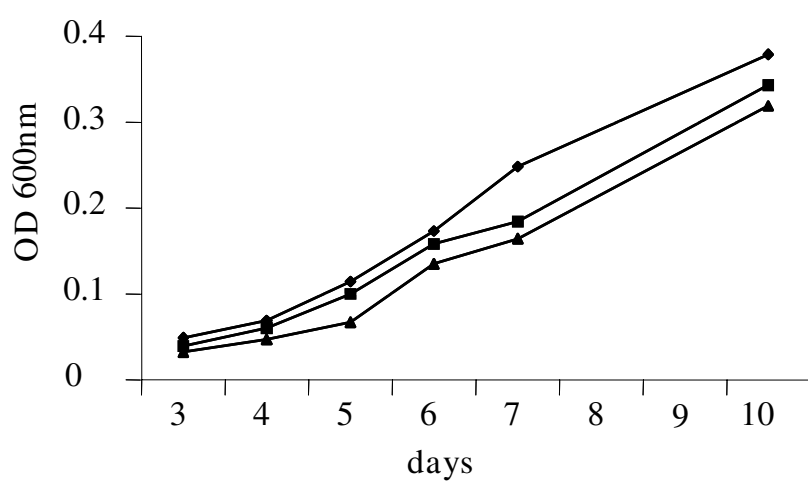

Figure 2

Effect of the mce3R mutation on in vitro growth of $M$. tuberculosis. Cultures of the mutant [ $\Delta$ mce $3 R$, square], the complemented [ $\Delta$ mce3R (pSummce3R), triangle] and the parental wild-type [H37Rv, rhombus] strains were grown to stationary phase and inoculated into fresh Dubos medium supplemented with $0.4 \%$ glucose at $O D_{600 \mathrm{~nm}} 0.005$ and the $O D_{600 \mathrm{~nm}}$ was measured at various time points. It is shown a representative experiment from triplicate. mce3 operon expression is repressed by Mce3R during in vitro culture and inside murine macrophages

We have previously demonstrated that the mce3 promoter allows the expression of the lacZ reporter gene in $M$. tuberculosis H37Rv but that this expression is completely abolished when Mce3R is overexpressed in the H37Rv strain from a multi-copy plasmid [13]. Although these findings constitute initial evidence demonstrating the role of Mce3R as a repressor of the mce3 operon transcription, the presence of an endogenous copy of the mce3R gene in $M$. tuberculosis did not enable us to determine the conditions in which the regulator system operates.

Here, in order to compare the expression the mce3 operon either in the absence or in the presence of Mce3R, DNA fusions of the mce3 promoter to lac $Z$ reporter, containing or not containing mce3R were cloned within pYUB178lac $Z$. The resulting plasmids, $\mathrm{pP} 3-\mathrm{mce} 3 \mathrm{R}$ and $\mathrm{pP} 3$ respectively, were integrated into the chromosome of the $\Delta$ mce3R strain. The $\beta$-galactosidase activity was measured at different points along cultures of $M$. tuberculosis grown in vitro and in a macrophages cell line. Since transcription of mce3 genes has previously shown to be increased when M. tuberculosis was grown in rich media [10-12] the expression of mce 3 operon was assessed in both synthetic (7H9) and rich (Dubos) media (Figure 3 and data not shown).

While hardly any $\beta$-galactosidase activity was detected either in in vitro cultures (Fig. 3) or in cell-line mice macrophages (Table 1) in the presence of Mce3R ( $\Delta$ mce3R::pP3-mce3R strain), the activity in the $\Delta$ mce3R::pP3 strain was remarkably high in all of the conditions tested. The promoter activity increased along the in vitro cultures of $\Delta$ mce3R:: pP3, and peaked at $24 \mathrm{~h}$ of infection inside the cell. These results clearly indicate that Mce3R represses the expression of mce3 operon in $M$. tuberculosis in the growth conditions tested.

\section{Assessment of the role of Mce3R in the transcription of the four mce operons}

A number of reports indicate that the expression of all mce genes depends on the growth conditions [10-12]. These observations, together with the findings that regulatory proteins are involved in the expression of the mce1 and mce 3 operons, suggested the idea of a broader regulatory mechanism differentially controlling the expression of mce genes. In order to test whether Mce3R is able to control the transcription of the other mce operons apart from mce3, the expression of one gene from each mce operon in the mutant strain was compared with that of the wild type. Primers were designed to amplify a 189, 168, 234, 151 and $134 \mathrm{bp}$ region on mce1D (Rv0172), mce $2 A$ (Rv0589), mce3E (Rv1970), mce4A (Rv3499), and sigA 


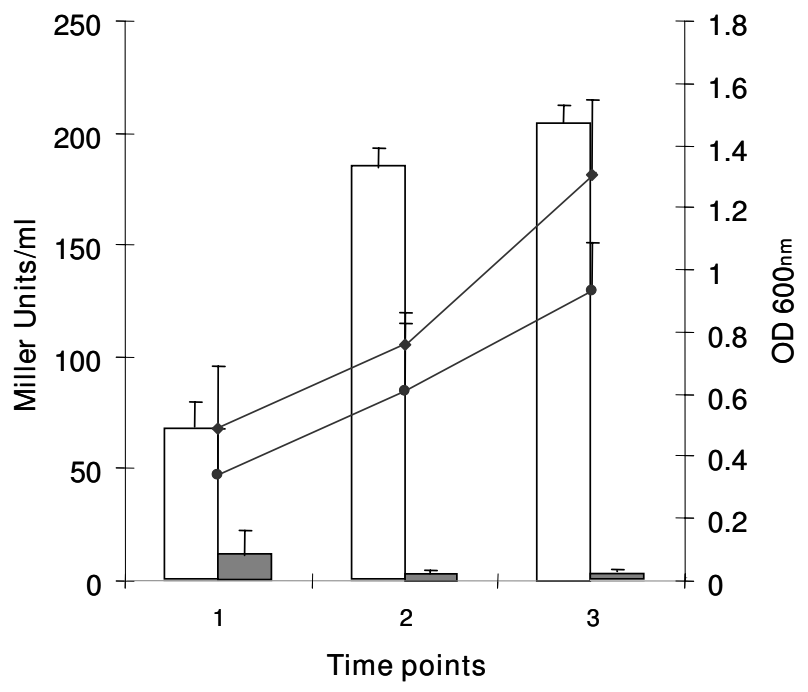

\section{Figure 3}

Effect of Mce3R on mce3 promoter activity during the growth of $\boldsymbol{M}$. tuberculosis. Comparison of mce 3 promoter activity during the growth of $\Delta$ mce3R (pP3-mce3RlacZ) (grey bars) and $\Delta$ mce3R (pP3-lacZ) (white bars) strains in M7H9-AD-G medium. The results are presented as $\beta$ galactosidase activity expressed as Miller units \pm S.D. of duplicate in three time points (I early exponential phase, 2 exponential phase and 3 stationary phase). Growth curves of $\Delta$ mce3R (pP3-lacZ) (square) and $\Delta$ mce3R (pP3-mce3RlacZ) (circle) strains are shown and the OD $600_{\mathrm{nm}}$ values are indicated on the right. Results represent one of at least three independent experiments.

respectively. Amplicons of expected size were obtained with each pair of primers (data not shown).

Differences in relative gene expression between the wild type and the mutant strains were assessed in group means for statistical significance by a randomisation test (see Materials and Methods). While the relative abundance of mce3E mRNA was significantly higher $(\mathrm{P}<0.030)$ in the mutant than in the wild type strain in the conditions evaluated, no significant differences between both strains were observed on the expression of mce1D $(\mathrm{P}<0.74)$, mce $2 A(\mathrm{P}<0.918)$ and mce4A $(\mathrm{P}<0.511)$ (Fig 4).
To verify that RNA samples were not contaminated with genomic DNA, RT-PCR reactions were performed without the addition of reverse transcriptase. The lack of amplification products verifies that the RT-PCR products were amplified from RNA that had been reversely transcribed into cDNA.

These results demonstrate that Mce3R regulates exclusively the transcription of mce3 operon among mce genes in the conditions tested

\section{Mce3R expression is self regulated}

To investigate whether $m c e 3 R$ is subject to transcriptional autoregulation, a transcriptional fusion was constructed between the $m c e 3 R$ promoter and the lac $Z$ reporter. Since $m c e 3 R$ is located adjacent to mce 3 operon and divergently oriented, the $m c e 3 R$ promoter is situated in mce3R-yrbE3A intergenic region. The entire intergenic region was fused to the lacZ gene within pYUB178-lacZ to create plasmid pPR3-lacZ. The pPR3-lacZ plasmid was transformed into the wild type M. tuberculosis H37Rv and the M. tuberculosis $\Delta$ mce3R mutant, and $\beta$-galactosidase activity was measured to assess the levels of mce3R promoter activity with and without Mce3R regulator. As shown in figure 5, in the presence of Mce3R (wild type H37Rv strain) the activity of $m c e 3 R$ promoter is steadily and significantly reduced as compared with that in the absence of the Mce3R regulator (mutant $\Delta$ mce3R strain). This reduction in mce3R promoter activity was more evident during the stationary growth phase. These experiments demonstrate that the Mce3R protein is able to transcriptionally repress expression of the $m c e 3 R$ promoter in $M$. tuberculosis during the in vitro culture condition tested.

\section{Discussion}

Little is known about gene regulation of virulence factors in $M$. tuberculosis due to its slow growth rate and the late development of mycobacterial genetics. Prokaryotic transcriptional regulators are classified in families on the basis of sequence similarity and structural and functional criteria. The TetR family, a family of transcriptional regulators that is well represented and widely distributed among bacteria, has a helix turn-helix (HTH) signature, the most recurrent DNA binding motif, to bind its target DNA. Members of the TetR family of repressors control transcription of proteins involved in multidrug resistance,

Table I: Effect of MceR on mce3 promoter activity in M. tuberculosis during infection of J774 Macrophage-like cell line.

\begin{tabular}{llll}
\hline & \multicolumn{2}{l}{$\beta$-galactosidase activityain J774 cell line } & \\
\hline Strain & $\mathbf{4 h}$ & $\mathbf{2 4} \mathbf{~ h}$ & $\mathbf{7 2 ~ \mathbf { ~ }}$ \\
$\Delta$ mce3R (pP3-mce3R) & 0 & 0 & 0 \\
$\Delta$ mce3R (pP3) & $114+/-5$ & $2571+/-172$ & $2162+/-92$
\end{tabular}

a calculated as: arbitrary $\beta$-galactosidase units/number of bacteria (CFU)/ 100000 .

Values are presented as the mean and standard deviation (SD) of reactions performed in triplicate. 


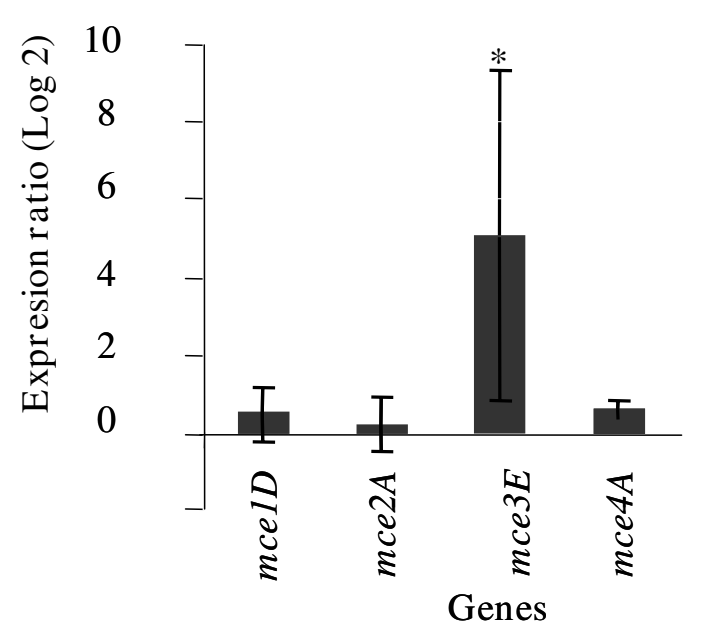

Figure 4

Effect of Mce3R on transcription of mce operons during in vitro growth of $M$. tuberculosis. The data are presented as the fold change in gene expression in mutant $\Delta$ mce3R strain normalized to sigA endogenous reference gene and relative to the wild type H37Rv strain +/- SD of derived results from four independent experiments. *Significant differences $(P<0.05)$ of gene expression in both strains as calculated by Pair Wise Fixed Reallocation Randomisation.

enzymes implicated in different catabolic pathways, biosynthesis of antibiotics, osmotic stress, and pathogenicity of gram-negative and gram-positive bacteria. At least 40 putative TetR-family regulator genes are spread on the $M$. tuberculosis genome. Most of them are similar to the TetR/ AcrR family, but just a few have been characterized. Mce3R was the first TetR-like regulator studied in M. tuberculosis [13]. Then, Engohang-Ndong et al. [22] found that a member of the TetR/CamR family represses the expression of ethA that encodes a protein that catalyses the activation of ethionamide (ETH). ETH is an important second-line anti TB drug used for the treatment of patients infected with multidrug-resistant strains.

The transcription profile of the mce3 operon in different in vitro growth conditions of $M$. tuberculosis has been addressed by RT-PCR in a number of publications and transcription of mce 3 genes has been found when bacteria were cultured both on LJ and Dubos media [11,12] but not in 7H9 synthetic medium in both exponential and stationary growth phases [10]. Using similar methodology we detected mRNA of mce3 operon during in vitro culture of M. tuberculosis either in synthetic or in rich (data not shown) media. However, transcription from the mce3 promoter, measured as $\beta$-galacosidase activity, was completely absent in the presence of Mce3R both in in vitro

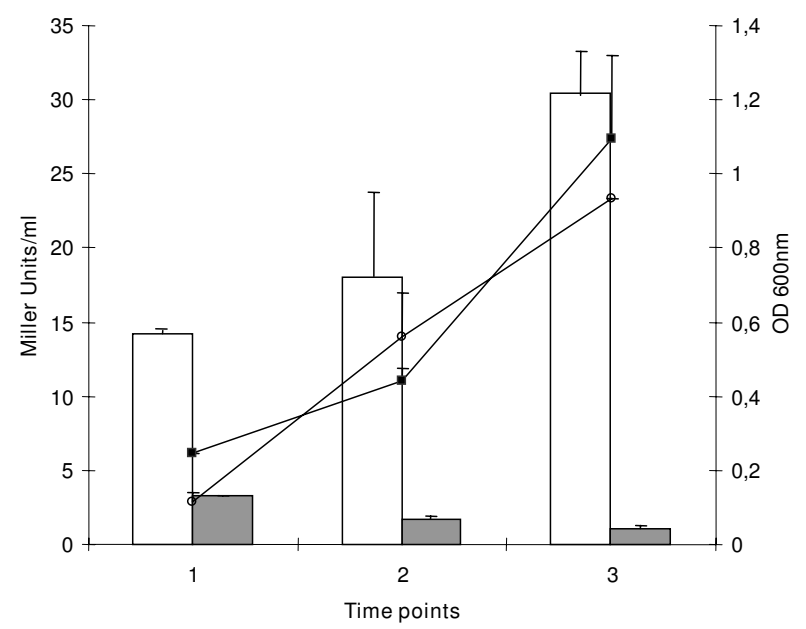

Figure 5

Effect of Mce3R on its own transcription during in vitro growth of $M$.tuberculosis. Comparison of $m c e 3 R$ promoter activity during the growth of $\Delta$ mce3R (PPR3-lacZ) (white bars) and H37Rv (pPR3-lacZ- Mce3R endogenous) (grey bars) strains in M7H9-AD-G medium. The results are presented as $\beta$-galactosidase activity expressed as Miller units \pm S.D. of duplicate in three time points (I early exponential phase, 2 exponential phase and 3 stationary phase). Growth curves of $\Delta$ mce3R (pPR3-lacZ) (circle) and H37Rv (pPR3lacZ- Mce $3 R$ endogenous) (rhombus) strains are shown and the OD $600_{n m}$ values are indicated on the right. Results represent one of three independent experiments.

conditions and inside a murine macrophages cell line. These last results, together with the finding that the elimination of Mce3R significantly increases mce3 transcription, indicate that the potential level of mce 3 expression is repressed in the conditions of growth assayed.

Interestingly, the high homology among mce operons is not conserved among their regulator genes, since Mce1 $\mathrm{R}$, the other mce regulator described, as well as the putative regulator of the mce 2 operon, belong to the GntR family $[9,1]$. In addition, no putative regulatory gene is placed in the vicinity of the mce 4 operon; however, it was recently proposed Rv3574, a TetR-type regulator, as represor of mce4 operon expression [23].

Here it was demonstrated that mce3R is not involved in the expression of mce1, mce 2 nor mce 4 operons. Therefore, it is tempting to speculate that both facts, i.e. gene redundancy and differential regulation, ensure the production of Mce proteins in different environments.

Here we demonstrated that mce3 promoter is stronger than $m c e 3 R$ promoter, and mce 3 expression seems to be 
mostly shut down during in vitro growth, but it is also likely that under unknown particular conditions of growth it would turn on.

As it happens to many other proteins of the TetR family, we found that Mce3R negatively regulates its own expression. The experiments with the mce3R promoter indicate that there is in average ten folds decreased in transcriptional activity when the Mce3R is provided. In this condition, the activity of mce3 promoter is sixty times repressed. Since the $m c e 3 R$ - yrbE3A intergenic region spans $880 \mathrm{bp}$, it is likely that Mce3R binds to consensus motifs located between the divergent genes in both promoter regions.

Although this study provides more insights to the role of Mce3R in the regulation of the mce operons, the information is still limited and further studies are necessary to detect any other gene regulated by this system. Elucidation of other promoters targeted by Mce3R will lead to the definition of a consensus mce3R-binding site and the possibility of define the Mce3R-regulon. Thus, one could hope to decipher the function of mce3 genes by the identification of the Mce3R regulon.

\section{Conclusion}

The available evidence demonstrates that while Mce3R represses powerfully the transcription of mce3 operon in vitro and inside macrophages, it does not affect the transcription of mce1, mce2 and mce4 operons during in vitro culture of $M$. tuberculosis. It was also demonstrated that Mce3R negatively regulates its own expression but the level of expression is lower than that observed for mce3 operon.

\section{Methods}

\section{Bacterial strains and culture media}

All cloning steps were performed in Escherichia coli DH5 $\alpha$. Regulation studies were performed in $M$. tuberculosis H37Rv. E. coli was grown in Luria-Bertani (LB) broth or on LB agar. M. tuberculosis strains were grown in Middlebrook 7H9 medium supplemented with $0.05 \%$ Tween 80 , Dubos and Middlebrook 7H11, all supplemented with albumin 0.5\%, dextrose $0.4 \%$ ), and $0.5 \%$ glycerol (M7H9-AD-G). When necessary, $20 \mu \mathrm{g}$ kanamycin $\mathrm{ml}^{-1}$ and $50 \mu \mathrm{g}$ hygromycin $\mathrm{ml}^{-1}$ were added to the media.

\section{General DNA methodology}

PCR amplifications from genomic DNA templates were performed as previously described [13]. Each primer contained base mismatches that introduced a restriction site suitable for directional cloning (Table 2). Chromosomal DNA samples were obtained as described vanSoolingen [24]. Purification of plasmids and DNA fragments were performed using the GFX Micro Plasmid Prep Kit (GE Healthcare) and DNA and Gel Band Purification Kit (GE
Table 2: Primer sequences used in this study.

\begin{tabular}{ll}
\hline Primer & Sequence* \\
\hline P3rev & ggatccggcgcggcgcaccagctggattcga \\
mce3R-P3up & ggatccggacacctcattcacaccgataatg \\
upMutReg & gcggccgcgagcgggaggtgaccaaggc \\
lowMutReg & gcggccgcgaagaaggccgacgcgaagc \\
upP3mce3Rint & tcatgaggacacctcattcacaccgataat \\
P3mce3Rrevint & tcatgaaccatggcgcggcgcaccagctggat \\
upP3int & aagcttttgcgcaccggaatcacaaatc \\
P3revint & aagcttaccatggcgcggcgcaccagctggat \\
mce3Eup & gacaccttcaccgcatacct \\
mce3Elow & ggtggtcttgttgaccgagt \\
sigAI & ggccagccgcgcacccttgac \\
sigA2 & gtccaggtagtcgcgcaggacc \\
mceIDup & ggcaagggtaagcaaatcaa \\
mceIDlow & ggtcaacctgtcggtgaact \\
mce2Aup & gaagaccgagctgactatgg \\
mce2Alow & atgtagcgaggattcacgtc \\
mce4Aup & ggtaggcaaggtcacggata \\
mce4Alow & aatgaattccaccgatttgg \\
P3anti up & aagcttggcgcggcgcaccagctgga \\
P3anti low & aagcttaccatttgcgcaccggaatcaca \\
\hline
\end{tabular}

* Restriction enzyme site added at the end of each primer is underlined.

Healthcare), respectively, according to the manufacturer's instructions. Plasmid pYUB178-lacZ was created by insertion of the $\beta$-galactosidase gene from plasmid pMC1871 (AmershamPharmacia) into HindIII and NheI sites of pYUB178 mycobacterial integrative vector [25]. M. tuberculosis H37Rv and M. tuberculosis $\Delta$ mce3R (see below) were transformed by electroporation, as described by Parish and Stoker [26].

\section{Construction of $M$. tuberculosis $\Delta$ mce $3 R$ mutant strain}

A genomic region containing $m c e 3 R$ and about $2 \mathrm{~kb}$ flanking $5^{\prime}$ and $3^{\prime}$ regions was obtained by PCR from M. tuberculosis H37Rv total DNA by using primers: upMutReg and lowMutReg. The amplified fragment was cloned in site NotI of p2NIL plasmid [21] and the mutant allele of $m c e 3 R$ was generated by inserting a cassette conferring hygromycin resistance from pUC-Hy7 (AmershamPharmacia) into a unique HindIII site internal to mce3R. The final delivery vector was generated by incorporation of the PacI cassette from pGOAL 17 into this last p2NIL recombinant vector. Mutants were constructed using a two-step strategy as described previously [21]. Chromosomal DNA was prepared from the selected clones and digested with EcoRI and then analyzed by Southern blotting by using the wild-type gene as probe. The mutant strain resulting from allelic exchange was designated $M$. tuberculosis $\Delta m c e 3 R$.

DNA fragment encompassing mce3R and the intergenic region between $m c e 3 R$ and $R v 1964$ was PCR amplified with primers: mce3R-P3up and P3rev and cloned into TOPO 2.1 vector (Invitrogen). A fragment containing 
$m c e 3 R$ and its promoter was released from this last plasmid by digestion with EcoRI and BamHI and cloned into pSUM41 [27] to produce plasmid pSummce3R. This plasmid was used to transform $M$. tuberculosis $\Delta$ mce3R strain by electroporation. The resulting complemented strain was referred to as $\Delta m c e 3 R:: m c e 3 R$.

\section{RNA preparation}

Culture pellets of $50 \mathrm{ml}$ were resuspended in $1 \mathrm{ml}$ of TRIzol (Invitrogen). Cells were disrupted using a Fastprep FP120 bead-beater (Savant) for $20 \mathrm{~s}$ at a speed of $6.0 \mathrm{~m} \mathrm{~s}^{-}$ ${ }^{1}$ with Lysing Matrix B (Q-Biogene). Then, $200 \mu$ l of chloroform (Merck) was added, and the mixture was incubated for $15 \mathrm{~min}$ at room temperature. Tubes were centrifuged at $10,000 \times g$ for $15 \mathrm{~min}$ at $4^{\circ} \mathrm{C}$, and the supernatant was extracted again with $100 \mu \mathrm{l}$ of chloroform and alcohol precipitated with $600 \mu \mathrm{l}$ of isopropanol and $60 \mu \mathrm{l}$ of $3 \mathrm{M}$ ammonium acetate $(\mathrm{pH} 5.3)$ at $-70^{\circ} \mathrm{C}$ overnight. Pellets were washed with $75 \%$ ethanol and resuspended in $50 \mu \mathrm{l}$ of diethyl pyrocarbonate-treated water (SigmaAldrich). The RNA preparations were treated with DNase amplified grade (Invitrogen).

\section{RT-Q-PCR}

DNA-free RNA ( $1 \mu \mathrm{g})$ extracted from middle logarithmicphase culture of either M. tuberculosis H37Rv or $\Delta$ mce3R was mixed with $50 \mathrm{ng}$ of random primers (Invitrogen) in $20 \mu \mathrm{l}$ of final volume and reversely transcribed to total cDNA with SuperScript III reverse Transcriptase (Invitrogen) following the manufacturer's instructions. Identical reactions lacking reverse transcriptase were also performed to confirm the absence of genomic DNA in all samples.

Q-PCR was performed in the Applied Biosystems 7000 DNA sequence detection system (Perkin-Elmer Corp.), by using Master Mix QuantiTect SYBR Green (Qiagen), $1 \mu \mathrm{l}$ of template cDNA and the pairs of primers listed on Table 1. Each reaction was performed in duplicate. Results were presented as ratios calculated with the Relative expression software tool (REST@) application described by Plaffl et al. [28]. Relative quantification of each target (mce) gene was performed by using sigA as reference gene and a subsequent test for significance of derived results was performed by using Pair Wise Fixed Reallocation Randomisation [29]. The value of PCR efficiency for all transcripts was 2 , as calculated following the formula: $\mathrm{E}=$ $10^{[-1 / \text { slope }] \text {. }}$

\section{Construction of $\beta$-galactosidase fusions}

DNA fragments encompassing the intergenic region between $m c e 3 R$ and operon mce3, either containing or not containing the coding sequence of mce $3 R$, were generated by PCR amplification with the pairs of primers upP3mce3Rint/P3mce3Rrevint and upP3int/P3revint, respectively. Both DNA fragments were cloned into the HindIII or the NcoI sites of p178-lacZ, giving rise to plasmids pP3-lacZ and pP3-mce3R-lacZ, respectively. The intergenic region was also amplified by PCR using primers P3antiup/P3antilow and the DNA fragment was cloned in plasmid pYUB178-lacZ to generate pPR3-lacZ. This last plasmid is the antisense version of pP3-lacZ in which the promoter of $m c e 3 R$ was fused to the reporter gene. These plasmids were used to transform $M$. tuberculosis strains as indicated.

\section{Measurements of $\beta$-galactosidase activity}

Determination of $\beta$-galactosidase activity in $M$. tuberculosis recombinant strains was performed as previously described [13]. Briefly, $\beta$-galactosidase activity was measured in soluble cell extract prepared from aliquots of in vitro cultures taken at different time points. Results were expressed in Miller units [A420 $\times 1000 /$ reaction time (min) xA600] [30].

Cultures of the murine macrophage-like cell line J774 were infected with recombinant $M$. tuberculosis $\mathrm{H} 37 \mathrm{Rv}$ strains (free of clumps) at a m.o.i. of 5. J774-infected cells were disrupted with $1 \%$ Triton $\times-100$, at 4,24 and $72 \mathrm{~h}$ post-infection. $\beta$-galactosidase measurements were performed on the soluble cell extract by using the Chemiluminescent lacZ $\beta$-galactosidase detection kit (MGT Product M08550) and the Luminometer, Veritas 1.4 (Turner Biosystems, Inc.). $\beta$-galactosidase activity was related to the number of bacteria as determined by bacterial counting.

\section{Authors' contributions}

MPS constructed the M. tuberculosis mutant strain and the plasmids, FCB performed the RT-QPCRs, MVB and OZ carried out cell infection experiments, AC participated in the design of the study and performed the statistical analysis, LK and FB conceived of the study, and participated in its design and coordination and drafted the manuscript. All authors read and approved of the final manuscript.

\section{Acknowledgements}

The present study was supported by SECyT grant Bid I728-OC/AR PICT 2002 - I I 765 and INTA grant AEBIO3454. A.C. and F.B. are CONICET fellows. L.K. is recipient of a fellowship from CONICET.

We thank Valeria Rocha for performing plasmids constructions, Teresa Moran for technical assistance in cell cultures, and Luis Fernandez for the bibliography provided. We also thank Dr. T. Parish for providing us with p2nil and pGOALI 7 plasmids.

\section{References}

I. Cole ST, Brosch R, Parkhill J, Garnier T, Churcher C, Harris D, Gordon SV, Eiglmeier K, Gas S, Barry CE III, Tekaia F, Badcock K, Basham D, Brown D, Chillingworth T, Connor R, Davies R, Devlin K, Feltwell T, Gentles S, Hamlin N, Holroyd S, Hornsby T, Jagels K, Krogh A, McLean J, Moule S, Murphy L, Oliver K, Osborne J, Quail MA, Rajandream M-A, Rogers J, Rutter S, Seeger K, Skelton J, Squares R, 
Squares S, Sulston JE, Taylor K, Whitehead S, Barrell BG: Deciphering the biology of Mycobacterium tuberculosis from the complete genome sequence. Nature 1998, 393:537-544.

2. Arruda S, Bonfim G, Knights R, Huima-Byron T, Riley LW: Cloning of an M. tuberculosis DNA fragment associated with entry and survival inside cells. Science |993, 26 I:| 454- | 457.

3. Flesselles B, Anand NN, Remani J, Loosmore SM, Klein MH: Disruption of the mycobacterial cell entry gene of Mycobacterium bovis BCG results in a mutant that exhibits a reduced invasiveness for epithelial cells. FEMS Microbiol Lett 1999, I 5:237-242.

4. Sassetti CM, Boyd DH, Rubin E): Genes required for mycobacterial growth defined by high density mutagenesis. Mol Microbiol 2002, 48:77-84.

5. Gioffre A, Infante E, Aguilar D, Santangelo MP, Klepp L, Amadio A, Meikle V, Etchechoury I, Romano MI, Cataldi A, Hernandez RP, Bigi F: Mutation in mce operons attenuates Mycobacterium tuberculosis virulence. Microbes Infect 2005, 7:325-334.

6. Shimono N, Morici L, Casali N, Cantrell S, Sidders B, Ehrt S, Riley LW Hypervirulent mutant of Mycobacterium tuberculosis resulting from disruption of the mceloperon. Proc Natl Acad Sci 2003, 100:15918-15923.

7. Chitale S, Ehrt S, Kawamura I, Fujimura T, Shimono N, Anand N, Lu S, Cohen-Gould L, Riley L: Recombinant Mycobacterium tuberculosis protein associated with mammalian cell entry. Cellular Microbiology 200I, 3:247-254.

8. Uchida Y, Casali N, White A, Morici L, Kendall LV, Riley LW: Accelerated immunopathological response of mice infected with Mycobacterium tuberculosis disrupted in the mcel operon negative transcriptional regulator. Cellular Microbiology 2007, 9:1275-83

9. Casali N, White AM, Riley LW: Regulation of the Mycobacterium tuberculosis mcel operon. J Bacteriol 2006, I88:44|-9.

10. Kumar M, Bose M, Brahmachari V: Analysis of Expression Profile of Mammalian Cell Entry (mce) Operons of Mycobacterium tuberculosis. Infect Immun 2003, 7 I:6083-6087.

II. Kumar A, Chandolia A, Chaudhry U, Brahmachari V, Bose M: Comparison of mammalian cell entry operons of mycobacteria: in silico analysis and expression profiling. FEMS Immunol Med Microbiol 2005, 43: 185-195.

12. Haile Y, Bjune G, Wiker HG: Expression of the mceA, esat-6 and hspX genes in Mycobacterium tuberculosis and their responses to aerobic conditions and to restricted oxygen supply. Microbiology 2002, 148:388I-3886.

13. Santangelo MP, Alito A, Caimi K, Zabal O, Zumarraga M, Romano MI: Cataldi, A, Bigi F: A negative regulator of mce3 operon in Mycobacterium tuberculosis. Microbiology 2002, I 48:2997-3006.

14. Ramos JL, Martinez-Bueno M, Molina-Henares AJ, Terán W, Watanabe K, Zhang X, Gallegos MT, Brennan R, Tobes R: The TetR Family of Transcriptional Repressors. Microbiology and Molecular Biology Reviews 2005, 69:326-356.

15. Chuanchuen R, Gaynor JB, Karkhoff-Schweizer R, Schweizer HP: Molecular Characterization of MexL, the Transcriptional Repressor of the mexJK Multidrug Efflux Operon in Pseudomonas aeruginosa. Antimicrobial Agents and Chemotherapy 2005, 49:|844-|85|.

16. Delany I, leva R, Alaimo C, Rappuoli R, Scarlato V: The iron-responsive regulator Fur is transcriptionally autoregulated and not essential in Neisseria meningitidis. J Bacteriol 2003, | 85:6032-604|.

17. Sala C, Forti F, di Florio E, Canneva F, Milano A, Riccardi G, Ghisotti $D$ : Mycobacterium tuberculosis FurA autoregulates its own expression. J Bacteriol 2003, 185:5357-5362.

18. Guilfoile PG, Hutchinson CR: The Streptomyces glaucescens TcmR protein represses transcription of the divergently oriented tcmR and tcmA genes by binding to an intergenic operator region. J Bacteriol 1992, 174:3659-3666.

19. Mc Murry L, Petrucci RE Jr, Levy SB: Active efflux of tetracycline encoded by four genetically different tetracycline resistance determinants in Escherichia coli. Proc Natl Acad Sci USA 1980, 77:3974-3977.

20. Orth P, Schnappinger D, Hillen W, Saenger W, Hinrichs W: Structural basis of gene regulation by the tetracycline inducible Tet repressor-operator system. Nat Struct Biol 2000, 7(3):2 I5-9.

21. Parish T, Stoker N: Use of a flexible cassette method to generate a double unmarked Mycobacterium tuberculosis tlyA
plcABC mutant by gene replacement. Microbiology 2000 | 46: 1969-1975.

22. Engohang-Ndong J, Baillat D, Aumercier M, Bellefontaine F, Besra G, Locht C, Baulard A: EthR, a repressor of the TetR/CamR family implicated in ethionamide resistance in mycobacteria, octamerizes cooperatively on its operator. Molecular Microbiology 2004, 5 I: | 75 - | 88

23. Kendall SL, Withers M, Soffair CN, Moreland NJ, Gurcha S, Sidders B, Frita R, Ten Bokum A, Besra GS, Lott JS, Stoker NG: A highly conserved transcriptional repressor controls a large regulon involved in lipid degradation in Mycobacterium smegmatis and Mycobacterium tuberculosis. Mol Microbiol 2007 65:684-99.

24. van Soolingen D, Hermans PWM, de Haas PEW, Soll DR, van Embden JDA: Occurrence and stability of insertion sequences in Mycobacterium tuberculosis complex strains: evaluation of an insertion sequence dependent DNA polymorphism as a tool in the epidemiology of tuberculosis. I Clin Microbiol I99|, 29:2578-2586.

25. Pascopella L, Collins FM, Martin JM Jr, Jacobs WR, Bloom BR: Identification of a genomic fragment of Mycobacterium tuberculosis responsible for in vivo growth advantage. Infect Agents Dis 1993, 2(4):282-284.

26. Parish T, Stoker NG: Electroporation of Mycobacteria. In Mycobacteria Protocols Volume 101. Method in Molecular Biology Humana Press Totowa, New Jersey; 1998: 120.

27. Aínsa JA, Martín C, Cabeza M, De la Cruz F, Mendiola MV: Construction of a family of Mycobacterium/Escherichia coli shuttle vectors derived from pAL5000 and pACYC 184: their use for cloning an antibiotic-resistance from Mycobacterium fortuitum. Gene 1996, 176:23-26.

28. Pfaffl MW, Horgan GW, Dempfle L: Relative expression software tool (REST) for group-wise comparison and statistical analysis of relative expression results in real-time PCR. Nucleic Acids Res 2002, 30:e36.

29. Rest gene-quantification [http://www.rest.gene-quantifica tion.info]

30. Miller JH: Experiments in Molecular Genetics Cold Spring Harbor, NY: Cold Spring Harbor Laboratory; 1972

Publish with Bio Med Central and every scientist can read your work free of charge

"BioMed Central will be the most significant development for disseminating the results of biomedical research in our lifetime. "

Sir Paul Nurse, Cancer Research UK

Your research papers will be:

- available free of charge to the entire biomedical community

- peer reviewed and published immediately upon acceptance

- cited in PubMed and archived on PubMed Central

- yours - you keep the copyright 